Article

\title{
Generalised Complex Geometry in Thermodynamical Fluctuation Theory
}

\author{
P. Fernández de Córdoba and J. M. Isidro * \\ Instituto Universitario de Matemática Pura y Aplicada, Universidad Politécnica de Valencia, \\ Valencia 46022, Spain; E-Mail: pfernandez@ mat.upv.es
}

* Author to whom correspondence should be addressed; E-Mail: joissan @ mat.upv.es.

Academic Editor: George Ruppeiner

Received: 29 May 2015 / Accepted: 19 August 2015 / Published: 20 August 2015

\begin{abstract}
We present a brief overview of some key concepts in the theory of generalized complex manifolds. This new geometry interpolates, so to speak, between symplectic geometry and complex geometry. As such it provides an ideal framework to analyze thermodynamical fluctuation theory in the presence of gravitational fields. To illustrate the usefulness of generalized complex geometry, we examine a simplified version of the Unruh effect: the thermalising effect of gravitational fields on the Schroedinger wavefunction.
\end{abstract}

Keywords: differential-geometric techniques; fluctuation theory; Unruh effect

\section{Introduction}

The theory of thermodynamical fluctuations provides a solid link between macroscopic and microscopic physics. Classical fluctuation theory [1] often sheds light on counterintuitive quantum-mechanical phenomena, thus helping to bridge the gap between the classical world and the quantum world. For example, Heisenberg's uncertainty principle can be nicely illustrated resorting to the theory of Gaussian fluctuations around thermal equilibrium [2].

On the other hand, the theory of thermodynamical fluctuations can be recast using the geometric language of differential manifolds [3-9]. This reexpression of a physical discipline in more abstract mathematical language goes a long way beyond a mere rewriting of the concepts involved. It renders the theory more versatile, enlarging its scope. Moreover, since the advent of Einstein's general relativity 
a century ago, (pseudo) Riemannian geometry belongs to the technical skills that any physicist has to master (at least at a working level). This places (pseudo) Riemannian geometry at a vantage point. In the opposite direction (i.e., thermodynamics as applied to geometry) one should mention at least two developments. The first one is a whole body of knowledge on the thermodynamics of black holes [10,11]. More recently, the reexpression of Einstein's relativity as a thermodynamics [12,13] has had far-reaching consequences for our understanding of spacetime.

Here we would like to report on another recent development in geometry with implications on the thermodynamics of fluctuations: the theory of generalized complex manifolds $[14,15]$.

In trying to understand the thorny relationship between gravity and the quantum [16-19] it has been argued that gravity acts dissipatively on quantum systems [20]. Specifically, in the presence of a gravitational field, thermal fluctuations become indistinguishable from quantum fluctuations [21-23]. This raises the fundamental question: How is one to treat thermal and quantum fluctuations on the same footing? Is it altogether possible? We will see here that generalized complex manifolds provide one viable answer to this question, one that appears not to have been explored yet in the geometrical approach to thermodynamics.

\section{Geometry and Fluctuations}

\subsection{Riemannian Geometry}

As a very elementary example, consider a thermodynamical system in an equilibrium state described by the following variables: temperature $T$, pressure $P$ and volume $V$. In the Gaussian approximation, choosing $T$ and $V$ as independent variables, the probability $W$ of a fluctuation $\Delta T, \Delta V$ around equilibrium is given by [24]

$$
W=W_{0} \exp \left[-\frac{C_{V}}{2 k_{B} T^{2}} \Delta T^{2}+\frac{1}{2 k_{B} T}\left(\frac{\partial P}{\partial V}\right)_{T} \Delta V^{2}\right]
$$

The thermodynamic inequalities $C_{V}>0$ and $(\partial P / \partial V)_{T}<0$ ensure that the argument of the above exponential is negative definite. This suggests considering the following (positive definite) Riemannian metric on the 2-dimensional manifold coordinatised by $T, V$ :

$$
\mathrm{d} s^{2}:=\frac{C_{V}}{2 k_{B} T^{2}} \mathrm{~d} T^{2}-\frac{1}{2 k_{B} T}\left(\frac{\partial P}{\partial V}\right)_{T} \mathrm{~d} V^{2}=: g_{i j} \mathrm{~d} x^{i} \mathrm{~d} x^{j}
$$

The metric coefficients $g_{i j}$ are of course $(T, V)$-dependent functions. This Riemannian structure encodes all the relevant information. For example, the average value $\langle f(T, V)\rangle$ of an arbitrary function $f=$ $f(T, V)$,

$$
\langle f(T, V)\rangle=Z^{-1} \int f(T, V) \exp \left(-g_{T T} T^{2}-g_{V V} V^{2}\right) \sqrt{g} \mathrm{~d} T \mathrm{~d} V
$$

where $Z:=\int \sqrt{g} \exp \left(-g_{T T} T^{2}-g_{V V} V^{2}\right) \mathrm{d} T \mathrm{~d} V$, naturally involves the metric. The role of Riemannian geometry in fluctuation theory is well known and has been reviewed at length in [8]. 


\subsection{Symplectic Geometry}

As our starting point here we will consider a certain thermodynamical system in equilibrium, in order to arrive at a corresponding symplectic structure.

Again in the Gaussian approximation, the probability $W$ of a fluctuation $\Delta P, \Delta V, \Delta T, \Delta S$ is given by [24]

$$
W=W_{0} \exp \left[-\frac{1}{2 k_{B} T}(-\Delta P \Delta V+\Delta T \Delta S)\right]
$$

Assume an equation of state $F(P, V, T)=0$ that can be solved for the temperature to obtain $T=g(P, V)$. For simplicity let us consider an ideal gas, $P V=S_{0} T$ :

$$
W=W_{0} \exp \left[-\frac{1}{2 k_{B}}\left(-S_{0} \frac{\Delta P \Delta V}{P V}+\frac{\Delta T \Delta S}{T}\right)\right]
$$

It is convenient to define the dimensionless variables

$$
p_{1}:=-\ln \left(\frac{P}{P_{0}}\right), \quad q_{1}:=\ln \left(\frac{V}{V_{0}}\right), \quad p_{2}:=\ln \left(\frac{T}{T_{0}}\right), \quad q_{2}:=\frac{S}{S_{0}}
$$

where $P_{0}, V_{0}$ and $T_{0}$ are reference values. Then Equation (5) becomes

$$
W=W_{0} \exp \left[-\frac{S_{0}}{2 k_{B}}\left(\Delta p_{1} \Delta q_{1}+\Delta p_{2} \Delta q_{2}\right)\right]
$$

We can regard $q_{1}$ and $q_{2}$ as coordinates on a thermodynamical configuration space $\mathbf{S}$, with $p_{1}$ and $p_{2}$ as their conjugate momenta. Thus the $q_{1}, p_{1}, q_{2}, p_{2}$ are Darboux coordinates for the symplectic form

$$
\omega=\mathrm{d} p_{1} \wedge \mathrm{d} q_{1}+\mathrm{d} p_{2} \wedge \mathrm{d} q_{2}
$$

In this way we identify $\Delta p_{1} \Delta q_{1}+\Delta p_{2} \Delta q_{2}$ in Equation (7) as the symplectic area of a 2-dimensional surface $\mathbf{F}$ induced by the fluctuation:

$$
\Delta p_{1} \Delta q_{1}+\Delta p_{2} \Delta q_{2}=\int_{\mathbf{F}}\left(\mathrm{d} p_{1} \wedge \mathrm{d} q_{1}+\mathrm{d} p_{2} \wedge \mathrm{d} q_{2}\right)
$$

Finally substituting Equation (9) into Equation (7) we find

$$
W=W_{0} \exp \left(-\frac{S_{0}}{2 k_{B}} \int_{\mathbf{F}} \omega\right)
$$

i.e., the probability of this thermal fluctuation is proportional to the exponential of the symplectic area of the fluctuation surface $\mathbf{F}$.

The importance of symplectic structures in classical mechanics is widely recognized and need hardly be recalled [25]. In fact not just Riemannian geometry, but also symplectic geometry, pertains to the realm of thermal fluctuations: the first law of thermodynamics endows the thermodynamic phase space with a contact structure, which includes symplectic geometry as a sub-case $[3,4,6,7]$.

A real $2 n$-dimensional manifold $\mathbf{M}$ is symplectic if there exists a closed, non-degenerate, rank 2 antisymmetric tensor field $\omega_{i j}$ defined everywhere on $\mathbf{M}$. Let $x^{i}$ be local coordinates around $x \in \mathbf{M}$, so $\omega=\frac{1}{2} \omega_{i j} \mathrm{~d} x^{i} \wedge \mathrm{d} x^{j}$ with $\omega_{j i}=-\omega_{i j}$. Since the matrix $\omega_{i j}$ is nonsingular, an inverse $\pi^{j k}$ exists such that 
$\omega_{i j} \pi^{j k}=\delta_{i}^{k}$. The Poisson brackets of two functions $f, g$ are defined as $\{f, g\}:=\pi^{j k} \partial_{j} f \partial_{k} g$, and the integrability condition $\mathrm{d} \omega=0$ turns out to be equivalent to the Jacobi identity for these Poisson brackets.

In this way the following symplectic analogue of Equation (3) allows one to compute the average value $\langle f\rangle$ of the function $f$ on $\mathbf{M}$ :

$$
\langle f\rangle=Z^{-1} \int_{\mathbf{M}} f \exp (-\omega)
$$

Above, the exponential $\mathrm{e}^{-\omega}$ is defined by Taylor expansion, powers being taken with respect to the wedge product. Then the $2 n$-dimensionality of the symplectic manifold picks out just one differential form that can be integrated against $\mathbf{M}$, namely the $2 n$-form $(-1)^{n} \omega^{n} / n !$; all other terms in the Taylor expansion give a vanishing contribution when integrated. The factor $(-1)^{n} / n$ ! has been included in the normalization $Z$. As had to be the case, this average involves the data concerning the symplectic structure on $\mathrm{M}$.

One can also regard a symplectic structure as providing an isomorphism from the tangent space $T_{x} \mathbf{M}$ into the cotangent space $T_{x}^{*} \mathbf{M}$ at each $x \in \mathbf{M}$. Specifically, the tangent vector $X=X^{i} \partial_{i}$ is mapped into the 1-form $\omega(X)=\xi=\xi_{i} \mathrm{~d} x^{i}$, with $\xi_{i}=\omega_{i j} X^{j}$. This viewpoint motivates the following definition (equivalent to the above, but more useful for later applications): a symplectic structure over a $2 n$-dimensional manifold $\mathbf{M}$ is an isomorphism $\omega_{x}$ between the tangent and the cotangent fibers over each point $x \in \mathbf{M}$,

$$
\omega_{x}: T_{x} \mathbf{M} \longrightarrow T_{x}^{*} \mathbf{M}
$$

such that, under the operation of taking the linear dual (denoted by an asterisk),

$$
\omega_{x}^{*}=-\omega_{x}, \quad \forall x \in \mathbf{M}
$$

Moreover, the integrability condition $\mathrm{d} \omega=0$ must be satisfied.

\subsection{Complex Geometry and Kähler Geometry}

Informally one could say that the imaginary unit is the hallmark of quantum mechanics. That $\mathrm{i}=\sqrt{-1}$ pertains to the quantum world has been very interestingly argued recently in [26,27]. More standard arguments have been known for long; such are the heat equation in imaginary time it, or the fact that quantum commutators $[\cdot, \cdot]$ formally equal $\sqrt{-1}$ times classical Poisson brackets $\{\cdot, \cdot\}$. Here we will briefly recall the role played by complex structures in the theory of coherent states [28,29].

Let $\mathbf{M}$ be a real $2 n$-dimensional phase space endowed with the symplectic form $\omega$. For simplicity let us also assume that $\mathbf{M}$ admits a holomorphic atlas compatible with the symplectic structure (this compatibility condition is called the Kähler property). In plain words, the real and imaginary parts of the holomorphic coordinates $z^{j}$ are Darboux coordinates for $\omega$ (here assumed dimensionless for simplicity):

$$
z^{j}=\frac{1}{\sqrt{2}}\left(q^{j}+\mathrm{i} p_{j}\right), \quad j=1, \ldots, n
$$

Upon quantisation, the Darboux coordinates $q^{j}$ and $p_{j}$ become operators $Q^{j}$ and $P_{j}$ on Hilbert space satisfying the Heisenberg algebra $\left[Q^{j}, P_{k}\right]=\mathrm{i} \delta_{k}^{j}$. Creation and annihilation operators are defined in the standard fashion: $A_{j}^{\dagger}:=\left(Q^{j}-\mathrm{i} P_{j}\right) / \sqrt{2}, A_{j}:=\left(Q^{j}+\mathrm{i} P_{j}\right) / \sqrt{2}$, and quantum excitations are measured 
with respect to a vacuum state $|0\rangle$ satisfying $A_{j}|0\rangle=0$, for all $j=1, \ldots, n$. Coherent states $\left|z^{j}\right\rangle$ are eigenvectors of $A_{j}$, the eigenvalues being the holomorphic coordinates (14):

$$
A_{j}\left|z^{j}\right\rangle=z^{j}\left|z^{j}\right\rangle, \quad j=1, \ldots, n
$$

(No sum over $j$ implied). In order to illustrate our point let us consider a 1-dimensional harmonic oscillator. The expectation value of the Hamiltonian operator $H=A^{\dagger} A+1 / 2$ in the state $|z\rangle$ equals $\langle z|H| z\rangle=|z|^{2}+1 / 2$. Since the energy fluctuation in the state $|z\rangle$ equals

$$
(\Delta H)_{z}=|z|, \quad z \in \mathbf{C}
$$

the relative fluctuation goes, for large enough $|z|$, like

$$
\frac{(\Delta H)_{z}}{\langle z|H| z\rangle} \simeq \frac{1}{|z|}, \quad|z| \rightarrow \infty
$$

But $1 /|z|$ is the inverse of the square root of the Kähler potential $K(z, \bar{z}):=|z|^{2}$ for the Euclidean metric on the complex plane $\mathrm{C}$. This simple example illustrates the important role played by complex manifolds in the quantum theory.

Every complex manifold $\mathbf{M}$ admits a (positive definite) Hermitian metric $h_{i j} \mathrm{~d} \bar{z}^{i} \mathrm{~d} z^{j}$ that is compatible with the complex structure [30]. Then an analogue of Equations (3) and (11) gives us the average value $\langle f\rangle$ of a function $f$ on $\mathbf{M}$ :

$$
\langle f\rangle=Z^{-1} \int_{\mathbf{M}} f \exp \left(-h_{i j} \bar{z}^{i} z^{j}\right) \sqrt{h} \prod_{k=1}^{n} \mathrm{~d} \bar{z}^{k} \wedge \mathrm{d} z^{k}
$$

The normalization $Z$ includes all factors of $\mathrm{i}=\sqrt{-1}$ coming from the volume element, and $h:=$ $\left|\operatorname{det} h_{i j}\right|$. As had to be the case, this average involves the data concerning the complex structure on M.

Formally, a complex structure $J$ over a real $2 n$-dimensional manifold $\mathbf{M}$ is an endomorphism of the tangent fibre over each point $x \in \mathbf{M}$

$$
J_{x}: T_{x} \mathbf{M} \longrightarrow T_{x} \mathbf{M}
$$

satisfying

$$
J_{x}^{2}=-\mathbf{1}, \quad \forall x \in \mathbf{M}
$$

as well as the integrability condition that the Nijenhuis tensor $N$ vanish identically. (We will not write down the Nijenhuis tensor explicitly; see reference [30] for details). Roughly speaking, Equation (20) expresses the existence of the imaginary unit $\mathrm{i}=\sqrt{-1}$ locally around the point $x \in \mathbf{M}$. The integrability condition $N=0$ ensures that the complex coordinates thus constructed locally truly transform holomorphically across different coordinate patches on the manifold M. (The Kähler property assumed in Equation (14) above is an additional hypothesis, that an arbitrary complex manifold may, but need not, satisfy in general). 


\subsection{Generalized Complex Geometry}

Our original motivation was the statement [21-23] that, in the presence of a gravitational field, quantum fluctuations become indistinguishable from thermal fluctuations. We have argued that thermal fluctuations are associated with symplectic structures, while quantum fluctuations come along with complex structures. How, then, is one to treat thermal and quantum fluctuations on the same footing? This is trivially achieved by those phase spaces $M$ that qualify as Kähler manifolds. However, the Kähler condition is very restrictive: not only does $M$ have to be simultaneously complex and symplectic; these two independent structures also have to be compatible.

In references. [3,4] the geometry of the thermodynamic phase space (including fluctuations) results in a para-Sasakian manifold, which is the contact-geometry equivalent of a Kähler manifold in symplectic geometry. This means that if one restricts to a proper even-dimensional subspace, the geometry is indeed that of a Kähler manifold. This geometry achieves the goal of treating thermal and quantum fluctuations on the same footing.

Generalized complex structures (GCS) also achieve the goal of providing a unified framework for thermal and quantum fluctuations. The following is a brief summary of GCS extracted from [14], duly tailored to meet our needs. For simplicity we prefer to work locally around a point $x \in \mathbf{M}$. Global issues can be taken care of by the corresponding integrability conditions, to be mentioned along the way whenever necessary. For our purposes the $2 n$-dimensional manifold $\mathbf{M}$ is assumed to be a phase space, that is, $\mathbf{M}=T^{*} \mathbf{S}$, for a certain $n$-dimensional configuration space $\mathbf{S}$.

Rather than considering the fibres $T_{x} \mathbf{M}$ or $T_{x}^{*} \mathbf{M}$ separately, in generalized complex geometry one considers their direct sum: over each point $x \in \mathbf{M}$ one erects the fibre $T_{x} \mathbf{M} \oplus T_{x}^{*} \mathbf{M}$. The total space of the bundle so constructed is $6 n$-dimensional: $2 n$ dimensions for the base $\mathbf{M}, 4 n$ dimensions for the fibre.

An inner product is defined on the fibre $T_{x} \mathbf{M} \oplus T_{x}^{*} \mathbf{M}$ :

$$
\langle X+\xi, Y+\eta\rangle:=\frac{1}{2}(\xi(Y)+\eta(X))
$$

Above, $X, Y \in T_{x} \mathbf{M}$ are tangent vectors, while $\xi, \eta \in T_{x}^{*} \mathbf{M}$ are 1-forms, all evaluated at $x \in \mathbf{M}$. It turns out that this inner product is pseudo-Riemann with signature $(2 n, 2 n)$. Hence the Lie group $S O(2 n, 2 n)$ acts on $T_{x} \mathbf{M} \oplus T_{x}^{*} \mathbf{M}$ by isometries. It is convenient to block-decompose the Lie algebra $s o(2 n, 2 n)$ as follows:

$$
\left(\begin{array}{cc}
A & \beta \\
B & -A^{*}
\end{array}\right)
$$

The diagonal blocks $A$ and $A^{*}$ are endomorphisms of their respective (sub)fibers, $A \in \operatorname{End}\left(T_{x} \mathbf{M}\right)$ and $A^{*} \in \operatorname{End}\left(T_{x}^{*} \mathbf{M}\right)$, while the offdiagonal blocks $B$ and $\beta$ connect these two (sub)fibers as per

$$
B: T_{x} \mathbf{M} \longrightarrow T_{x}^{*} \mathbf{M}, \quad \beta: T_{x}^{*} \mathbf{M} \longrightarrow T_{x} \mathbf{M}
$$

Moreover, upon taking the dual we have $B^{*}=-B, \beta^{*}=-\beta$. This antisymmetry allows us to regard the block $B$ as a 2 -form in $\Lambda^{2} T_{x}^{*} \mathbf{M}$ if we set

$$
B(X)=i_{X} B
$$

For illustrative purposes let us express Equation (24) in local coordinates $x^{i}$ around a point $x \in \mathbf{M}$, so $B$ becomes the matrix $B_{i j}$. Given the vector $X=X^{j} \partial_{j} \in T_{x} \mathbf{M}$, the object $i_{X} B$ is defined to be the 
covector whose components are $B_{i j} X^{j} \in T_{x}^{*} \mathrm{M}$. We see that this is exactly the way a symplectic form $\omega$ behaves. Since $\omega$ can be regarded as an element of $\Lambda^{2} T_{x}^{*} \mathbf{M}$, so can $B$. (Contrary to $\omega$, however, $B$ need neither be closed nor non-degenerate).

The particular isometries of the fibre $T_{x} \mathbf{M} \oplus T_{x}^{*} \mathbf{M}$ obtained by setting $A=0=\beta$ in Equation (22) and exponentiating,

$$
\exp \left(\begin{array}{cc}
0 & 0 \\
B & 0
\end{array}\right)=\left(\begin{array}{cc}
\mathbf{1} & 0 \\
B & \mathbf{1}
\end{array}\right)
$$

are the pseudo-orthogonal transformations

$$
X+\xi \longrightarrow X+\xi+i_{X} B
$$

The isometries (26), called $B$-transformations, will play an important role.

A generalized complex structure over $\mathbf{M}$, denoted $\mathcal{J}$, is an endomorphism of the fibre over each $x \in \mathbf{M}$,

$$
\mathcal{J}_{x}: T_{x} \mathbf{M} \oplus T_{x}^{*} \mathbf{M} \longrightarrow T_{x} \mathbf{M} \oplus T_{x}^{*} \mathbf{M}
$$

such that the following two conditions hold. First,

$$
\mathcal{J}_{x}^{2}=-\mathbf{1}, \quad \forall x \in \mathbf{M}
$$

Second,

$$
\mathcal{J}_{x}^{*}=-\mathcal{J}_{x}, \quad \forall x \in \mathbf{M}
$$

The above two conditions are formulated locally around any $x \in \mathbf{M}$; as usual they need not be compatible with changes of coordinate charts on $\mathrm{M}$. The Courant integrability condition, whose validity we will henceforth assume without stating its contents explicitly, ensures this compatibility; see [14,15] for details.

Comparing now Equations (29) and (13), we are led to the particular case when $\mathcal{J}$ at $x \in \mathbf{M}$ is given by

$$
\mathcal{J}_{\omega_{x}}=\left(\begin{array}{cc}
0 & -\omega_{x}^{-1} \\
\omega_{x} & 0
\end{array}\right)
$$

where $\omega$ is a symplectic form. One says that this $\mathcal{J}_{\omega}$ defines a GCS of symplectic type.

Similarly, the comparison of Equations (28) and (20) suggests the particular case of a GCS given by

$$
\mathcal{J}_{J_{x}}=\left(\begin{array}{cc}
-J_{x} & 0 \\
0 & J_{x}^{*}
\end{array}\right)
$$

where $J$ is a complex structure. We say that the above $\mathcal{J}_{J}$ defines a GCS of complex type.

Furthermore, GCS succeed at interpolating between the above opposite types, the symplectic type and the complex type; let us explain this more carefully. A point $x \in \mathbf{M}$ is said to be regular if it possesses a neighborhood $\mathcal{N}_{x}$ on which there exists a Poisson structure $\omega^{-1}$ with constant rank. In a neighborhood $\mathcal{N}_{x}$ of any regular point $x \in \mathbf{M}$ one can define a diffeomorphism and a $B$-transformation, the combined action of which maps $\mathcal{N}_{x}$ into the product $\mathcal{C}_{x} \times \mathcal{R}_{x} \subset \mathbf{C}^{k} \times \mathbf{R}^{2 n-2 k}$. Here $\mathcal{C}_{x}$ is an open set within the standard complex manifold $\mathbf{C}^{k}$, and $\mathcal{R}_{x}$ is an open set within the standard symplectic manifold $\mathbf{R}^{2 n-2 k}$. 
The nonnegative integer $k$ is called the type of the GCS $\mathcal{J}$, the limiting cases of Equations (30) and (31) respectively corresponding to $k=0$ and $k=n$. As described in [14,15], the type $k$ need not be constant across $\mathrm{M}$ : it may vary from one point to another in $\mathbf{M}$.

In plain words, any generalized complex manifold factorizes locally as the product of a complex manifold times a symplectic manifold.

Finally assume that $\mathbf{M}$ is a linear space. Then any generalized complex structure of type $k=0$ is the $B$-transform of a symplectic structure. This means that any generalized complex structure of type $k=0$ can be written as

$$
\mathrm{e}^{-B} \mathcal{J}_{\omega} \mathrm{e}^{B}=\left(\begin{array}{cc}
-\omega^{-1} B & -\omega^{-1} \\
\omega+B \omega^{-1} B & B \omega^{-1}
\end{array}\right)
$$

for a certain 2-form $B$; use has been made of Equations (25) and (30). Similarly any generalized complex structure of type $k=n$ over a linear manifold $\mathbf{M}$ is the $B$-transform of a complex structure,

$$
\mathrm{e}^{-B} \mathcal{J}_{J} \mathrm{e}^{B}=\left(\begin{array}{cc}
-J & 0 \\
B J+J^{*} B & J^{*}
\end{array}\right)
$$

after using Equations (25) and (31). When $\mathbf{M}$ is an arbitrary smooth manifold, not necessarily a linear space, statements (32) and (33) remain basically true, with some minor modifications required; see references $[14,15]$ for details.

The consequences of the above become immediately apparent. Let us for simplicity assume that the type $k$ is constant across $\mathbf{M}$. Then any GCS with an extremal value of $k$, i.e., either $k=0$ or $k=n$, can always be reduced to the corresponding canonical form (30) or (31) by means of a $B$-transformation. Thus $k=0$ corresponds to a thermal description of phenomena, while $k=n$ corresponds to a quantum description of phenomena, no interpolation existing between the two descriptions. Nonextremal values of the type, i.e., such that $0 \neq k \neq n$, contain both thermal and quantum descriptions simultaneously.

Average values $\langle f\rangle$ of functions $f$ on generalized complex manifolds are defined by an obvious modification of the product of the right-hand sides of Equations (11) and (18).

\section{When "Quantum" Becomes "Thermal"}

Any gravitational field is locally equivalent to an accelerated frame. In an accelerated frame, quantum becomes thermal; this is basically the content of the Unruh effect [31] (in an admittedly lax formulation that is however precise enough for our purposes). Without using the full apparatus of relativistic quantum field theory, let us see how quantum can become thermal in the simplified setup of the quantum mechanics of a nonrelativistic particle. This understood, we will analyse the role played by the GCS on phase space under the passage from an inertial frame to an accelerated frame. We will conclude that the transformation law for the Schroedinger wavefunction under the passage to a noninertial frame (as in the Unruh effect) is governed by a $B$-transformation of the GCS on phase space.

A remark is in order. The gravitational field considered here must be weak in order to rule out effects such as, e.g., relativistic speeds, or the likely breakdown of standard quantum mechanics in the presence of very strong gravitational fields [20]. Such phenomena lie beyond our scope. 


\subsection{Inclusion of a Gravitational Field}

In flat Euclidean space $\mathbf{R}^{3}$, let $K$ denote an inertial frame with origin $O$ and axes $O x, O y$ and $O z$. Let $K^{\prime}$ denote a uniformly accelerated frame, with origin $O^{\prime}$ and axes $O^{\prime} x^{\prime}, O^{\prime} y^{\prime}$ and $O^{\prime} z^{\prime}$ respectively parallel to $O x, O y$ and $O z$. For simplicity we will assume that, at $t=0$, the two origins $O$ and $O^{\prime}$ coincide, their relative velocity also vanishing at $t=0$. Let the acceleration $\vec{\alpha}$ of $K^{\prime}$ with respect to $K$ be $(\alpha, 0,0)$, with $\alpha$ a constant. Coordinates $(x, y, z)$ with respect to $K$ are related to coordinates $\left(x^{\prime}, y^{\prime}, z^{\prime}\right)$ with respect to $K^{\prime}$ as per

$$
x=x^{\prime}+\frac{1}{2} \alpha t^{2}, \quad y=y^{\prime}, \quad z=z^{\prime}, \quad t=t^{\prime}
$$

We consider a point particle of mass $m$ fixed to the origin $O^{\prime}$, thus at rest with respect to $K^{\prime}$. If $H$ denotes the Hamiltonian of the particle as seen from the inertial frame $K$, then the Hamiltonian $H^{\prime}$ in $K^{\prime}$ reads

$$
H^{\prime}=H-p_{x} \alpha t+\frac{m}{2} \alpha^{2} t^{2}
$$

with the momenta $p_{x}$ and $p_{x}^{\prime}$ related as per $p_{x}^{\prime}=p_{x}-m \alpha t$. In the inertial frame $K$ we have a Schroedinger equation $\mathrm{i} \hbar \partial \psi / \partial t=H \psi$. Our aim is to derive a transformation law for the wavefunction $\psi$ such that, in the accelerated frame $K^{\prime}$, the Schroedinger equation will read i $\hbar \partial \psi^{\prime} / \partial t=H^{\prime} \psi^{\prime}$. For this purpose let us make the Ansatz

$$
\psi^{\prime}=\psi \exp [f(t)]
$$

$f(t)$ being an undetermined function of the time variable. In this way we arrive at the following differential equation for the unknown function $f$ :

$$
\mathrm{i} \hbar \frac{\mathrm{d} f}{\mathrm{~d} t}=-p_{x} \alpha t+\frac{1}{2} m \alpha^{2} t^{2}
$$

Dropping an irrelevant integration constant and substituting the result into Equation (36) leads to

$$
\psi^{\prime}=\exp \left[-\frac{\mathrm{i}}{\hbar}\left(\frac{1}{6} m \alpha^{2} t^{3}-\frac{1}{2} p_{x} \alpha t^{2}\right)\right] \psi
$$

Clasically, the particle is at rest in the frame $K^{\prime}$, so $p_{x}^{\prime}=0$ implies $p_{x}=m \alpha t$. Quantum-mechanically we can only state that the centre of mass remains at rest at $x^{\prime}=0$, the wavepacket spreading around this average position. With this understanding we can also set $\left\langle p_{x}\right\rangle=p_{x}=m \alpha t$ in Equation (38). We conclude that, taking the wavefunction in the accelerated frame to be

$$
\psi^{\prime}=\exp \left(\frac{\mathrm{i}}{\hbar} \frac{1}{3} m \alpha^{2} t^{3}\right) \psi
$$

ensures the form invariance of the Schroedinger equation under the transformation from an inertial frame to an accelerated frame. For time lapses that are short enough, and/or for accelerations that are weak enough, the speeds attained will never become relativistic. Within this limited range, Newtonian mechanics (and its quantum counterpart, the Schroedinger equation) can be trusted. 


\subsection{The Unruh Effect}

The next step is to invoke de Broglie [32] in order to write an inverse proportionality between time $t$ and temperature $T$ :

$$
-\frac{\mathrm{i}}{t}=\frac{k_{B}}{\hbar} T
$$

Thus substituting Equation (40) into Equation (39) we find

$$
\psi^{\prime}=\exp \left(-\frac{1}{3} \frac{m \alpha^{2} \hbar^{2}}{k_{B}^{3} T^{3}}\right) \psi .
$$

Moreover, from the above we can read off what power law must relate the acceleration to the temperature of the accelerated frame: $\alpha$ must be proportional to $T$, while dimensional analysis provides the necessary conversion factors. Specifically,

$$
\alpha=2 \pi \frac{c k_{B}}{\hbar} T
$$

The dimensionless normalization factor $2 \pi$, that cannot be derived using our simplified treatment, comes from a full quantum-field-theoretical analysis [31]. Finally substituting Equation (42) into Equation (41) we arrive at

$$
\psi^{\prime}=\exp \left(-\frac{4 \pi^{2}}{3} \frac{m c^{2}}{k_{B} T}\right) \psi
$$

Equations (43) and (39) are equivalent, the equivalence between the two being guaranteed by the de Broglie relation Equation (40) and the Unruh relation Equation (42).

The Boltzmann-like factor present in Equation (43) bears out the fact that the effect of the gravitational field on the Schroedinger wavefunction is of thermal nature. Due to the assumptions made in our derivation, Equation (43) is valid only for intermediate temperatures. The limit $T \rightarrow \infty$ is excluded (because this would require strong gravitational fields); so is the limit $T \rightarrow 0$ (because of the inverse proportionality Equation (40) between time and temperature).

\subsection{Transformation to an Accelerated Frame as a B-Transformation}

Classical phase space is spanned by the coordinates $x, y, z$ and their conjugate momenta $p_{x}, p_{y}, p_{z}$. For the rest of the discussion, the dimensions $y, p_{y}, z, p_{z}$ can be ignored, as they are unaffected by the change of frame Equation (34). Thus, for our purposes, the manifold $\mathbf{M}$ of Section 2.4 can be taken to be that subspace of classical phase space spanned by $x$ and $p_{x}$, i.e., $\mathbf{R}^{2}$.

Now the manifold $\mathbf{R}^{2}$ can be endowed with a GCS. This can be done in two equivalent ways. One can consider the GCS of complex type defined on $\mathbf{R}^{2}=\mathbf{C}$ by the complex coordinates Equation (14). Alternatively, one can consider the GCS of symplectic type defined on $\mathbf{R}^{2}$ by the standard symplectic form $\omega=\mathrm{d} x \wedge \mathrm{d} p_{x} / \hbar$. Since our interest lies in considering the effect of $B$-transformations, and $\mathbf{R}^{2}=\mathbf{C}$ is a Kähler manifold, the type of the CGS considered is immaterial.

We claim that the transformation law for the Schroedinger wavefunction under the passage to an accelerated frame, Equation (39), follows from a $B$-transformation of the GCS on phase space $\mathbf{R}^{2}$, Equation (26). In other words, the Schroedinger wavefunction keeps track of which frame is being used, the bookkeeping device being the GCS on phase space. Verifying that such is indeed the case requires, so to speak, translating the geometer's language into the physicist's language. This we do next. 
Tangent vectors $X$ at the point $\left(x, p_{x}\right) \in \mathbf{R}^{2}$ are objects

$$
X=a \partial_{x}+b \partial_{p_{x}} \in T_{\left(x, p_{x}\right)} \mathbf{R}^{2}, \quad a, b \in \mathbf{R}
$$

Similarly, tangent covectors $\xi$ at the point $\left(x, p_{x}\right) \in \mathbf{R}^{2}$ are objects

$$
\xi=c \mathrm{~d} x+d \mathrm{~d} p_{x} \in T_{\left(x, p_{x}\right)}^{*} \mathbf{R}^{2}, \quad c, d \in \mathbf{R}
$$

As the basepoint $\left(x, p_{x}\right) \in \mathbf{R}^{2}$ is moved around, we obtain a vector field $X$ and a field of differentia l-forms $\xi$ on $\mathbf{R}^{2}$. This amounts to promoting the numbers $a, b, c, d$ to real-valued functions $a\left(x, p_{x}\right)$, $b\left(x, p_{x}\right), c\left(x, p_{x}\right), d\left(x, p_{x}\right)$ on $\mathbf{R}^{2}$. Finally, an object such as $X+\xi$ in Equation (26) is the direct sum of a vector field and a field of differential 1-forms on $\mathbf{R}^{2}$-a section of the direct sum bundle $T \mathbf{R}^{2} \oplus T^{*} \mathbf{R}^{2}$.

Next we reexpress the $B$-transformation (26) as the variation

$$
\delta(X+\xi)=\delta X+\delta \xi=\delta \xi=i_{X} B
$$

Above we have used the fact that, under a $B$-transformation, $X$ remains unchanged. The $B$-field is a 2-form on $\mathbf{R}^{2}$,

$$
B=B\left(x, p_{x}\right) \mathrm{d} x \wedge \mathrm{d} p_{x}
$$

with a certain coefficient function $B\left(x, p_{x}\right)$. Now

$$
\delta \xi=i_{X} B=a\left(x, p_{x}\right) B\left(x, p_{x}\right) \mathrm{d} p_{x}+b\left(x, p_{x}\right) B\left(x, p_{x}\right) \mathrm{d} x
$$

The above is a 1-form field, so it can be added to $X+\xi$ as required by Equation (26). Let us now make the following specific choice for the vector field $X$ :

$$
a\left(x, p_{x}\right)=x, \quad b\left(x, p_{x}\right)=p_{x}
$$

In the physicist's language, this $X$ is just the position vector on phase space $\mathbf{R}^{2}$. Substituted into Equation (48), this choice for $X$ yields

$$
\delta \xi=i_{X} B=x B\left(x, p_{x}\right) \mathrm{d} p_{x}+p_{x} B\left(x, p_{x}\right) \mathrm{d} x
$$

Along the motion of the particle located at $O^{\prime}$ we can write, using Equation (34),

$$
\mathrm{d} p_{x}=m \alpha \mathrm{d} t, \quad \mathrm{~d} x=\alpha t \mathrm{~d} t
$$

Substitution of Equations (34) and (51) into (50) leads to

$$
\delta \xi=i_{X} B=\frac{3}{2} B\left(x(t), p_{x}(t)\right) m \alpha^{2} t^{2} \mathrm{~d} t
$$

The above is a 1-form, that can be integrated along the trajectory followed by the particle between $\tau=0$ and $\tau=t$. We denote by $\Delta \xi(t)$ the number so obtained:

$$
\Delta \xi(t):=\int_{0}^{t} \delta \xi=\frac{3}{2} m \alpha^{2} \int_{0}^{t} B\left(x(\tau), p_{x}(\tau)\right) \tau^{2} \mathrm{~d} \tau
$$


When $B$ is a constant, the integral can be evaluated explicitly:

$$
\Delta \xi(t)=\frac{1}{2} B m \alpha^{2} t^{3}
$$

That the function $B\left(x(t), p_{x}(t)\right)$ is actually constant on $\mathbf{R}^{2}$ implies that the 2-form $B$ in Equation (47) becomes a mere scalar multiple of the canonical symplectic form on phase space. Specifically, picking $B=2 / 3$ we find in Equation (54)

$$
\Delta \xi(t)=\frac{1}{3} m \alpha^{2} t^{3}
$$

The right-hand side of the above equals ( $-\mathrm{i} \hbar$ times) the argument of the exponential in the Unruh transformation law Equation (39). Therefore the latter can be reexpressed as

$$
\psi^{\prime}=\exp \left(\frac{\mathrm{i}}{\hbar} \Delta \xi(t)\right) \psi
$$

Summarising, we may say that the Unruh effect acts on the wavefunction by multiplication with the exponential of (i/ $\hbar$ times) the integral of a $B$-field along the particle's trajectory on phase space. The vector field $X$ involved in this $B$-transformation is just the position vector on phase space, while the $B$-field considered is a mere scalar multiple of the canonical symplectic form on phase space.

\subsection{A Nonuniform Gravitational Field}

The relation just derived between the Unruh effect and the $B$-transformation of the GCS on phase space was based on the assumption that the gravitational field was static and spatially constant. In turn, this assumption made it possible to choose a constant $B$-field on phase space (actually a scalar multiple of the symplectic form). A nonstatic and/or nonuniform gravitational field can be mimicked by a nonstatic and/or nonuniform acceleration vector $\vec{\alpha}$. This lends plausibility to the following hypothesis:

Hypothesis 1. Regard classical phase space as a generalized complex manifold. In the presence of a nonstatic and/or nonuniform, but nevertheless weak, gravitational field, the inertial-frame Schroedinger wavefunction $\psi$ remains form-invariant under a transformation to a locally accelerated frame, where its value is $\psi^{\prime}$, provided that $\psi$ and $\psi^{\prime}$ are related according to the law

$$
\psi^{\prime}=\exp \left(\frac{\mathrm{i}}{\hbar} \Delta \xi(t)\right) \psi
$$

Above,

$$
\Delta \xi(t):=\int_{0}^{t} i_{X} B\left(x(\tau), p_{x}(\tau)\right) \mathrm{d} \tau
$$

is a line integral along the particle's trajectory in phase space, while $X$ is the position vector of the particle along the said trajectory. Moreover, whenever the generalised complex structure on classical phase phase is of symplectic type, the 2-form B is an appropriate scalar multiple of the symplectic form $\omega$.

We defer analysis of the above hypothesis for further study. 


\section{Conclusions}

We have presented a brief review of some recent developments in differential geometry with applications to thermodynamical fluctuation theory. Standard wisdom draws a clear frontier between thermal fluctuations and quantum fluctuations. While this separation is perfectly consistent in the absence of gravitational fields, this border becomes fuzzy in the presence of gravity [20-23]. A well-known example of this mixing is the Unruh effect $[31,33,34]$. Another instance of a gravitational incursion into the thermal realm is the Ehrenfest-Tolman effect [35]. One can expect an eventual theory of quantum gravity to enhance, rather than diminish, this mixing of thermal and quantum phenomena.

In this article we have examined the thermalising effect of weak, classical gravitational fields on the Schroedinger wavefunction from the point of view of generalised complex geometry on classical phase space. Using the transformation law for the Schroedinger wavefunction under the passage to an accelerated frame, we have derived the nonrelativistic Unruh effect. As expected, the latter establishes a linear dependence law between the acceleration of the noninertial frame and the temperature thereby generated. Within the scope of the techniques presented here lie other interesting physical systems, to be treated in an upcoming publication. Such are quantum-classical hybrids [36,37] and the thermalising properties of nonuniform (but still weak and classical) gravitational fields.

Altogether, we conclude that generalised complex geometry provides a powerful tool to analyse fluctuation theory and thermal phenomena in the presence of gravity.

\section{Author Contributions}

Both authors contributed equally to this article. Both authors have read and approved the final manuscript.

\section{Conflicts of Interest}

The authors declare no conflict of interest.

\section{References}

1. Callen, H. Thermodynamics; Wiley: New York, NY, USA, 1960.

2. Velazquez, L. Principles of Classical Statistical Mechanics: A Perspective from the Notion of Complementarity. Ann. Phys. 2012, 327, 1682-1693.

3. Bravetti, A; Lopez-Monsalvo, C. Para-Sasakian Geometry in Thermodynamic Fluctuation Theory. J. Phys. A 2015, 48, 125206.

4. Bravetti, A; Lopez-Monsalvo, C.S.; Nettel, F. Contact Symmetries and Hamiltonian Thermodynamics. Ann. Phys. 2015, 361, 377-400.

5. Quevedo, H.; Vázquez, A. The Geometry of Thermodynamics. AIP Conf. Proc. 2008, 977, 165, doi:10.1063/1.2902782.

6. Rajeev, S. Quantization of Contact Manifolds and Thermodynamics. Ann. Phys. 2008, 323, 768-782.

7. Rajeev, S. A Hamilton-Jacobi Formalism for Thermodynamics. Ann. Phys. 2008, 323, 2265-2285. 
8. Ruppeiner, G. Riemannian Geometry in Thermodynamic Fluctuation Theory. Rev. Mod. Phys. 1995, 67, 605.

9. Velazquez, L. Curvature of Fluctuation Geometry and its Implications on Riemannian Fluctuation Theory. J. Phys. A 2013, 46, 345003.

10. Bardeen, J.M.; Carter, B.; Hawking, S.W. The Four Laws of Black Hole Mechanics. Comm. Math. Phys. 1973, 31, 161-170.

11. Ruppeiner, G. Thermodynamic Curvature and Black Holes. In Breaking of Supersymmetry and Ultraviolet Divergences in Extended Supergravity, Proceedings of the INFN-Laboratori Nazionali di Frascati School 2013; Springer: Switzerland, 2014; Springer Proceedings in Physics, Volume 153; pp. 179-203.

12. Padmanabhan, T. Thermodynamical Aspects of Gravity: New Insights. Rept. Prog. Phys. 2010, 73, 046901.

13. Padmanabhan, T. General Relativity from a Thermodynamic Perspective. Gen. Rel. Grav. 2014, 46, 1673.

14. Gualtieri, M. Generalized Complex Geometry. Ann. Math.. 2011, 174, 75-123.

15. Hitchin, N. Generalized Calabi-Yau Manifolds. Q. J. Math. 2003, 54, 281-308.

16. Calmet, X. Quantum Mechanics, Gravity and Modified Quantization Relations. High Energy Phys. Theory 2015, doi: 10.1098/rsta.2014.0244.

17. Elze, H.-T. Are Nonlinear Discrete Cellular Automata Compatible with Quantum Mechanics? Quantum Phys. 2015, 631, 012069.

18. 'T Hooft, G. The Cellular Automaton Interpretation of Quantum Mechanics. A View on the Quantum Nature of our Universe, Compulsory or Impossible? 2015, arXiv:1405.1548.

19. Matone, M. Equivalence Postulate and Quantum Origin of Gravitation. Found. Phys. Lett. 2002, 15, 311-328.

20. Penrose, R. The Road to Reality: A Complete Guide to the Laws of the Universe; Alfred A. Knopf: London, UK, 2004.

21. Kolekar, S; Padmanabhan, T. Indistinguishability of Thermal and Quantum Fluctuations. 2013, arXiv:1308.6289.

22. Smolin, L. On the Nature of Quantum Fluctuations and their Relation to Gravitation and the Principle of Inertia. Class. Quant. Grav. 1986, 3, 347, doi:10.1088/0264-9381/3/3/009.

23. Smolin, L. Quantum Gravity and the Statistical Interpretation of Quantum Mechanics. Int. J. Theor. Phys. 1986, 25, 215-238.

24. Landau, L; Lifshitz, E. Statistical Physics, Part 1; Pergamon Press: Oxford, UK, 1980.

25. Arnold, V. Mathematical Methods of Classical Mechanics; Springer: Berlin, Germany, 1989.

26. Kauffman, L. Eigenforms and Quantum Physics. Cybern. Human Knowing. V. 2011, 18, 111-121.

27. Kauffman, L. Iterants, Fermions and the Dirac Equation. 2014, arXiv:1406.1929.

28. Florentino, C.; Matias, P.; Mourão J.; Nunes, J.P. Geometric Quantization, Complex Structures and the Coherent State Transform. 2005, arXiv:math/0402313.

29. Perelomov, A. Generalized Coherent States and their Applications; Springer: Berlin, Germany, 1986. 
30. Kobayashi, S; Nomizu, K. Foundations of Differential Geometry; Wiley: New York, NY, USA, 1996.

31. Unruh, W. Notes on Black-Hole Evaporation. Phys. Rev. D 1976, 14, 870.

32. De Broglie, L. La thermodynamique «Cachée» des particules. Ann. Inst. Henri Poincaré 1964, 1, 1-19. Available online: https://drive.google.com/file/d/0B0xb4crOvCgTemNOektLV25Kc0U/edit (accessed on 20 August 2015).

33. Davies, P. Scalar Production in Schwarzschild and Rindler Metrics. J. Phys. A 1975, 8, 609.

34. Fulling, S. Nonuniqueness of Canonical Field Quantization in Riemannian Space-Time. Phys. Rev. D 1973, 7, 2850.

35. Tolman, R. Relativity, Thermodynamics and Cosmology; Dover: New York, NY, USA, 1987.

36. Elze, H.-T. Linear Dynamics of Quantum-Classical Hybrids. Phys. Rev. A 2012, 85, 052109.

37. Elze, H.-T. Action Principle for Cellular Automata and the Linearity of Quantum Mechanics. Phys. Rev. A 2014, 89, 012111.

(c) 2015 by the authors; licensee MDPI, Basel, Switzerland. This article is an open access article distributed under the terms and conditions of the Creative Commons Attribution license (http://creativecommons.org/licenses/by/4.0/). 